\title{
Volunteer Project Competition Impact Research on Volunteer Organization-Based on the influence of China Youth Volunteer Service Project Competition on a university in Wuhan
}

\author{
Mingyue Yin ${ }^{1, a,{ }^{*}}$, Ziqing $\mathrm{Li}^{2, \mathrm{~b}}$ and Dila $\mathrm{A}^{3, \mathrm{c}}$ \\ 1,2 School of Management, Wuhan University of Technology, Wuhan, Hubei, China \\ ${ }^{3}$ School of Safety and Emergency Management, Wuhan University of Technology, Wuhan, Hubei, \\ China \\ ayinmingyue9840@126.com, b2890719543@qq.com, c1556306640@qq.com
}

Keywords: University management; Volunteer service; Questionnaire.

\begin{abstract}
At present, the development of volunteer organizations in major universities in China is booming, and volunteer activities have become an integral part of the life of college students. A number of volunteer service competitions are held each year to encourage more young people to join the volunteer service, such as the China Youth Volunteer Service Competition. The main participants of these competitions are volunteer organizations of various universities. Participating in such competitions has become an important activity of various volunteer organizations. This paper, through a questionnaire survey of volunteers who participated in the 4th China Youth Volunteer Service Competition, believes that a certain degree of such volunteer project competition is beneficial to the competition. The promotion and innovation of volunteer organizations, but too many competitions will occupy more staff distribution of volunteer organizations.
\end{abstract}

\section{志愿者项目比赛对志愿者组织影响研究 一一基于中国青年志愿服务项目大赛对武汉某高校影响 \\ 尹明月 $1, a,{ }^{*}$ ，李子情 $2, b$, 阿迪拉 ${ }^{3, c}$ \\ 1,2 武汉理工大学管理学院, 武汉, 湖北, 中国 \\ 3 武汉理工大学安全与应急管理学院, 武汉, 湖北, 中国 ayinmignyue9840@126.com, b2890719543@qq.com, c1556306640@qq.com}

关键词：高校管理; 志愿服务; 问卷调查

中文摘要. 目前我国各大高校的志愿者组织发展欣欣向荣, 志愿活动已成为了高校学生的生 活组成部分。每年会举办诸多志愿服务类比赛, 鼓励号召更多青年加入志愿服务中去, 如中 国青年志愿服务项目大赛, 这些比赛的主要参与者为各高校的志愿者组织。参与此类比赛已 经成为了各类志愿者组织的重要活动, 本文通过对于参加过第四届中国青年志愿服务项目大 赛的志愿者进行问卷调查, 认为一定程度上的此类志愿者项目比赛有利于志愿者组织的提升 和创新，但是过多的比赛又会占用志愿者组织较多的人员分配。 


\section{1. 引言}

目前高校中众多的志愿者组织已成为现代大学的重要组成部分, 志愿活动也成为大学生 提升自我、服务社会的重要方式。与此同时，志愿者类比赛也出现在各省各高校。志愿者类 比赛的初衷是鼓励更多人参与志愿活动，加强不同志愿者群体之间的交流，提升志愿服务水 平。但是另一方面, 众多的志愿者类比赛也让各个志愿者组织不得不抽出足够的人员参与此 类比赛, 因此志愿者类比赛对于志愿者组织的影响程度如何成为了一个有待于研究的问题, 本文以志愿者领域重要的国家级比赛——中国青年志愿项目服务大赛对于武汉某高校的影响 进行研究。

\section{2. 中国青年志愿项目服务大赛}

中国青年志愿项目服务大赛是由共青团中央、中央文明办、民政部、中国残联、中国志 愿服务联合会、中国青年志愿者协会以及大赛决赛所在地政府等多家机构公共主办的大型全 国志愿者赛事 ${ }^{11]}$ 。笔者所在的学校连续四届参与此比赛。

\section{3. 调查与分析}

本文对武汉某高校参与过第四届中国青年志愿服务大赛的志愿者组织进行调研，共发放 问卷 107 份, 其中回收有效问卷98份, 回收率为91.5\%。问卷共设置7项问题，分别从志愿者项 目服务大赛对于个人及组织的影响, 进行正、反两方面考察。问卷采用 spss 20.0 进行数据分析, 问卷总体质量水平较高。分析结果表明 Cronbach $\alpha$ 系数为 0.821 , 问卷的信度可接受。问卷效 度分析采用因子分析测得KMO值为 0.855 , 效度较高。本问卷参照李克特五级量表设置, 1-5 分别表示该项陈述符合自己认识的程度，得分越高表示越认同。问卷回收结果如表1:

表1 问卷回收结果

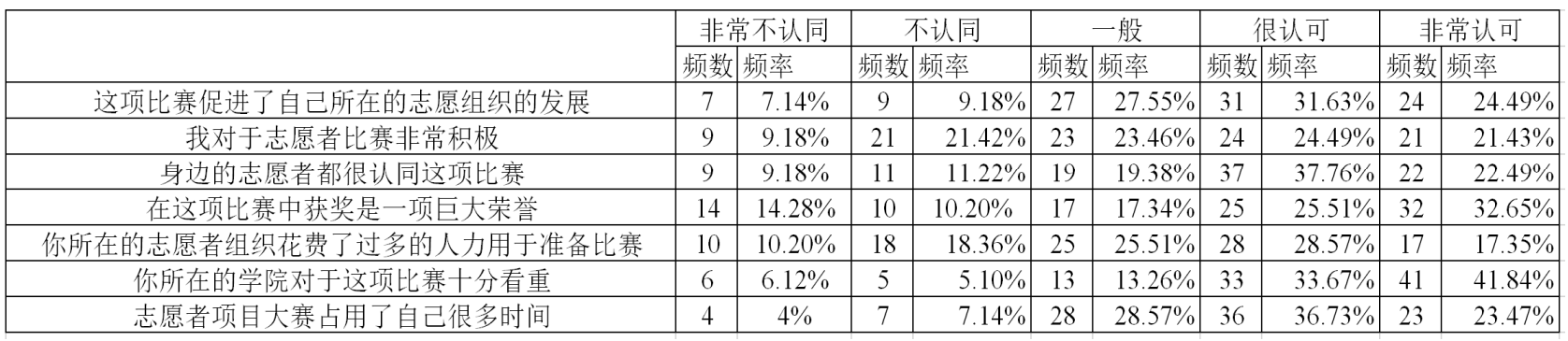

\section{1 个人访谈}

笔者首先访问了几位志愿者组织的负责人，负责人对志愿者比赛给组织的影响说法差别 较大。有的负责人认为, 比赛有利于志愿项目的成熟化。特别是全国性比赛, 能够了解优秀 的项目, 开拓志愿者的视野, 也使志愿者通过接触专业性的赛事, 在志愿项目筹划、展示等 方面获得锻炼, 使志愿者自身与组织得到提升。但部分负责人认为当前各级志愿类比赛较多, 高校内不仅每年有定期举办的比赛, 而且会为国、省级赛事展开系列选拔赛。因此过多的赛 事消耗了志愿者组织的人力资源, 又由于比赛所获荣誉受到重视, 志愿者组织会承担一定的 压力, 所以志愿者类比赛对于志愿者组织产生了一定的不利影响。基于上述访谈, 笔者进行 了较大范围的问卷发放。通过问卷分析, 得出志愿者项目大赛对于志愿者组织的影响情况。

\section{2 问卷分析}

（1）志愿者项目大赛促进志愿者组织的发展 
从图1可以看出, 98名受访对象中, 认为志愿者项目大赛对于志愿者组织发展的起到促进 作用的占大多数。其中“很认可”、“非常认可”占总数的 $56.1 \%$, 因此可以认为大部分志愿者对 于志愿者项目大赛持支持态度, 认为比赛对志愿者组织的提升起到了正面作用。

志愿者项目大赛促进志愿者组织的发展

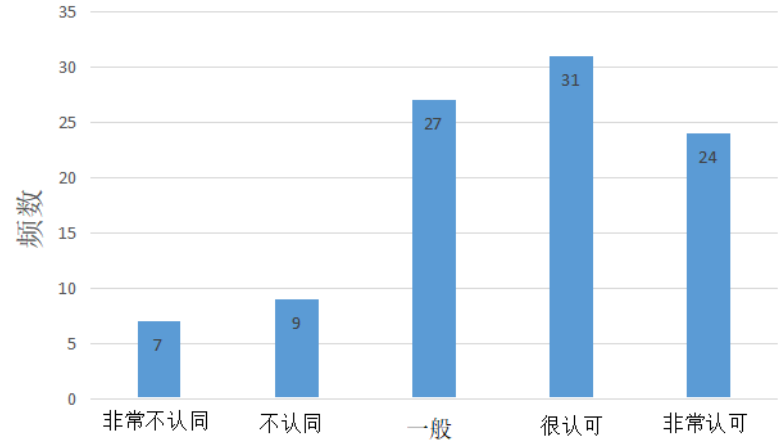

图1 志愿者项目大赛促进志愿组织的发展认可度
我对于志愿者比赛非常积极

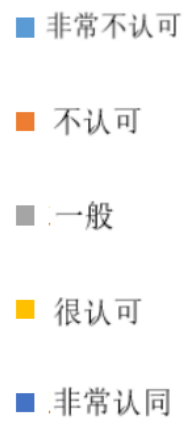

图2 我对于志愿者比赛非常积极

（2）我对于志愿者比赛非常积极

由结果可看出，“很认可”和“非常认可”共 57 人，约占总人数的 $58.2 \%$, 因此可以认为大部 分志愿者对于志愿类比赛持积极态度。但“非常不认可”和“不认可”的人仍占到了 $30.6 \%$, 也可 以认为在志愿者项目大赛的筹备过程中, 部分志愿者组织的内部可能存在一定的不合理现象。 因此各志愿者组织应该优化筹备过程，提升志愿者在筹备比赛过程中的积极性。

(3) 身边的志愿者都很认同这项比赛

如图3，受访对象选择最多的是“很认可”，其次是“非常认可”，由于本问题所考量的是基 于志愿者个人周边的人际关系中对于志愿者项目大赛的看法, 由此可以认为, 认同志愿项目 大赛的志愿者数量较多, 这也与所调查的学校长期对志愿者项目大赛的正面宣传有关。在实 际调研中发现, 在参加志愿者比赛的组织中, 只有部分同学作为筹备人员且与该项赛事有直 接关系, 更大范围内的志愿者并未直接参与, 但是从问卷回收结果中可以看出, 大范围内的 志愿者对于该项赛事持有正面认同的态度。

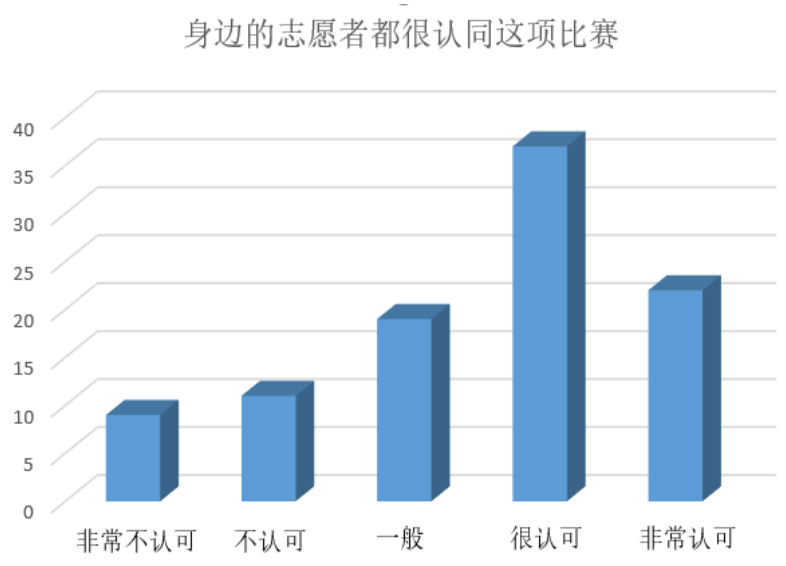

图3 身边的志愿者都很认同这项比赛

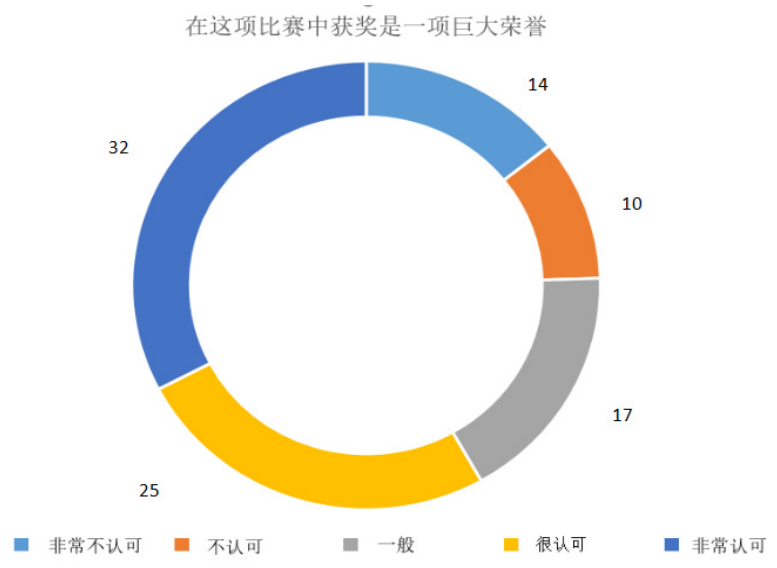

图4 在这项比赛中获奖是一项巨大的荣誉

（4）在这项比赛中获奖是一项巨大的荣誉

从问卷回收分析的情况来看（图4），认同“在这项比赛中获奖是一项巨大的荣誉”的受访 者数量为 57 人, 占比为 $58.16 \%$ 。符合上述相似问题中考核的问题, 因此可以认为, 从受访者 个人价值观念来说，对于志愿者项目大赛的认可程度较高。

(5) 两项从负面影响考核的指标

在问卷中设置两项从负面影响进行考察的问题, 分别为“你所在的志愿者组织花费了过多 的人力用于准备比赛”、“志愿者项目大赛占用了自己很多时间”, 数据分析结果如下图: 


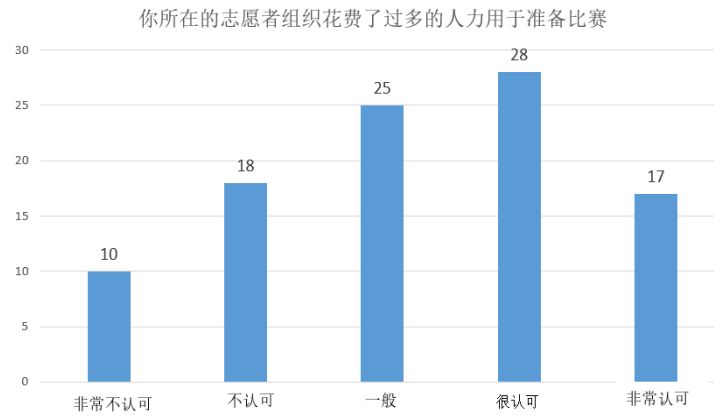

图5 志愿者组织是否花费过多的人力准备比赛

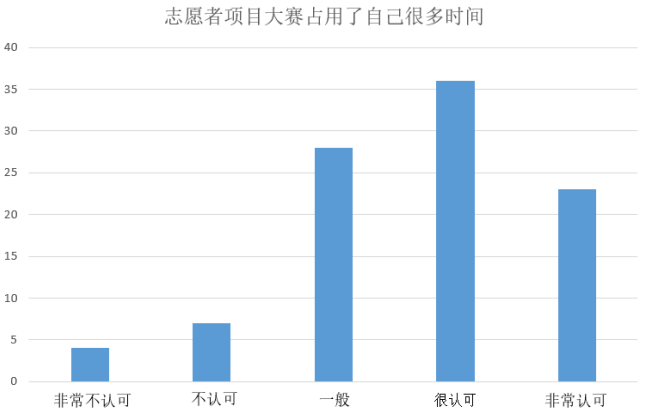

图6 志愿者项目大赛占用了自己很多时间

在前期的调查中，笔者通过访谈了解到比赛对于志愿者群体影响在于筹备大赛耗费过多 的人力, 影响了组织其他活动的开展。问卷从志愿者组织和个人两个层面出发, 考察同一类 问题。从数据上看, 两项问题中选择“很认可”或“非常认可”的受访者均超过半数, 因此可认定 之前调查中发现的大赛一定程度上过多占用了志愿者组织的人力这一结论的正确性。

(6) 你所在的学院对于这项比赛十分看重

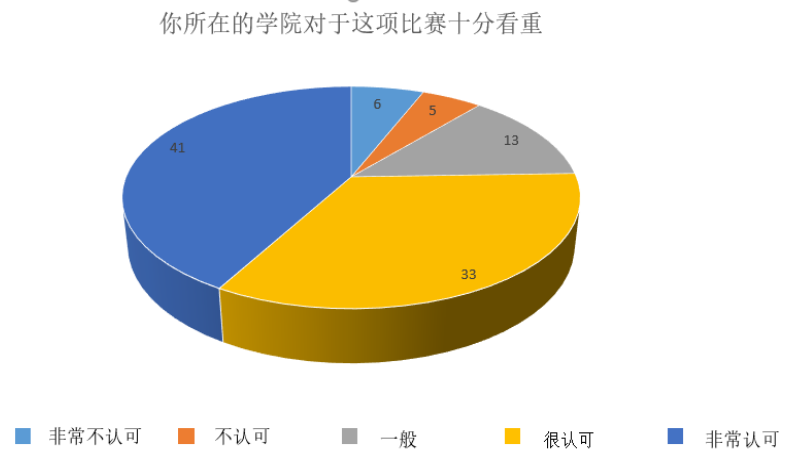

图7 你所在的学院对于这项比赛十分看重

目前我国高校大部分志愿者组织隶属于团委。通过访谈了解到志愿者组织的负责人在比 赛的筹备上受领导及指导老师的影响很大, 这也与目前高校学生组织管理模式有重要关系。 从回收结果来看，选择“很认可”以及“非常认可”的受访者达到 74 人，占总数的 $75.5 \%$ 。因此可 以看出, 在志愿者组织对于项目大赛的筹备过程中, 整个组织传达了对志愿者项目大赛的重 视，因此这样的压力传导也让绝大多数志愿者感同身受。

\section{4. 总结}

本文通过深入访谈法和问卷调查法对武汉某高校志愿者对中国青年志愿服务项目大赛的 态度进行了综合考察。从普通志愿者角度分析得到比赛对于高校志愿者组织有促进作用, 也 造成了负担。该项比赛给全国的志愿者团队提供了交流的机会，有助于各组织整体提升。但 为筹备比赛要组成专门的小组, 占用了组织的人力资源, 又因受各方重视, 进一步加大了对 比赛的资源投入倾斜。因此组织应合理安排筹备比赛的人力, 不应过多影响正常活动的开展。

\section{References}

[1] Qingping Pu, Nan Zhao, et al. "Research on the Influence of Volunteer Service on Volunteer Political Identity__Based on the Big Data Survey of National Volunteer Service Project Competition" Journal of Chongqing University (Social Science Edition) Vol. 23, No. 2, 2017 129-137.

[2] Can Li, Ling Xin, "Research on the Evaluation Method of Reliability and Validity of Questionnaires", China Health Statistics, Vol. 25, No. 5, October 2008 541-544. 